\title{
L'usage des langues minoritaires dans la production des émissions télévisuelles : une approche comparative des cas breton, irlandais et māori
}

The use of minority languages in television production: a comparative study of the cases of Breton, Irish and Maori

\section{Ruth Lysaght}

\section{OpenEdition}

\section{Journals}

Édition électronique

URL : https://journals.openedition.org//bl/1043

ISSN : 2727-9383

\section{Éditeur}

Université de Bretagne Occidentale - UBO

Édition imprimée

Date de publication : 1 juin 2015

Pagination : 55-80

ISBN : 979-10-92331-16-5

ISSN : 1270-2412

\section{Référence électronique}

Ruth Lysaght, "L'usage des langues minoritaires dans la production des émissions télévisuelles : une approche comparative des cas breton, irlandais et māori », La Bretagne Linguistique [En ligne], 19 | 2015, mis en ligne le 01 mai 2021, consulté le 22 mai 2021. URL : http://journals.openedition.org/lbl/ 1043 
Ruth LYSAGHT*

\section{L'usage des langues minoritaires dans la production des émissions télévisuelles : une approche comparative des cas breton, irlandais et māori}

\section{L'}

influence des médias sur la pratique des langues minoritaires n'a toujours pas été complétement étudiée (Fishman, 2001: 473; Cormack, 2007). Si son pouvoir par rapport au public reste difficile à mesurer, il est plus évident d'examiner comment le monde des médias peut créer ou non un environnement où la langue minorisée est parlée entre collègues sur le lieu de travail.

Cet article vise à comparer les pratiques des producteurs/réalisateurs et des diffuseurs des médias contemporains en irlandais, māori et breton. Ces pratiques sont nées de l'actualité des paysages audiovisuels et reflètent aussi la philosophie identitaire des acteurs. Or l'image de la langue ne réside pas seulement dans les programmes diffusés, mais aussi dans les esprits et les pratiques des équipes qui les réalisent. On peut dire que les émissions représentent la trace de la langue pendant le processus de production.

Bien sûr, les aléas de la production, ainsi que le contexte sociolinguistique actuel, grandit le défi des réalisateurs. Comment ces

* Docteur en études des médias, University of Auckland, Nouvelle-Zélande (travaille actuellement à l'Université de Bretagne occidentale). 
acteurs vivent-ils et conçoivent-ils leur situation? Une approche comparative permet de mettre en évidence la nature généralisée du dilemme. Puis on analysera quelques approches institutionnelles et particulières dans le but d'identifier des stratégies pour surmonter ces défis communs.

Mon enquête vise à mettre en lumière la façon dont les personnes qui travaillent dans le secteur de production perçoivent leur entreprise et leur rôle au sein de celle-ci, tout en tenant compte de la politique linguistique inhérente au projet. Les questions-clés traitées dans cet article révèlent des défis de production en langue minorisée aujourd'hui, le contenu des émissions, la vision du public qu'ont mes interlocuteurs, leurs conditions de travail et leurs espoirs pour un meilleur avenir ${ }^{1}$.

\section{Contexte scientifique}

La plupart des travaux universitaires traitant des langues minorisées et de la télévision sont éclairés par les idées de Mike Cormack. Cormack a développé l'idée de 'Minority Language Media Studies'/ Études des Médias de langue minoritaire (MLM) en tant que champ distinct de recherche, et il a étudié la situation en Europe de l'ouest, surtout en Écosse. MLM est conçu comme trait d'union entre les études linguistiques, sociologiques, géopolitiques, culturelles et des médias. Dans un livre paru en 2007, coédité avec Niamh Hourigan, Cormack regroupe les essais de plusieurs chercheurs concernant les médias en Europe (Cormack \& Hourigan, 2007). Hourigan indique notamment les différents rapports de force et le niveau de soutien des États aux langues régionales et aux langues parlées par les immigrés.

Dans le cadre français, plusieurs auteurs ont exploré les repré-

1. J'ai travaillé sur des émissions pour des sociétés de production indépendantes dans les trois pays concernés, et j'ai également assisté à d'autres tournages, à l'extérieur et en studio. Cette expérience m'a donné une perspective détaillée des enjeux. En plus de cela, au cours de ma recherche, j'ai effectué plus de 50 entretiens avec des acteurs des médias en breton, en maori et en irlandais (journalistes, responsables, monteurs, réalisateurs, directeurs...) qui travaillent dans des radios associatives et nationales, des chaînes de télévision ou plateformes internet ainsi que des représentants publics. Cet article fournira un premier aperçu d'un champ d'enquête jusqu'ici peu labouré et je sollicite les commentaires et les critiques (ruth.lysaght@gmail.com). 
sentations des minorités sur l'écran. On trouve dans un livre édité par Isabelle Rigoni (2007) un chapitre de Jacques Guyot (Guyot, 2007 (qui a également écrit un autre article sur MLM en général, 2007)), qui s'intéresse à la situation des langues régionales en France. En ce qui concerne la langue bretonne, il existe bien des articles et ouvrages sur des éléments sociolinguistiques, historiques et littéraires (Broudic, 2009; Calvez, 2011 ; Le Coadic, 2002 ; Le Pipec, 2012). Ces œuvres m'ont permis d'aborder le sujet et de mieux connaître le contexte dans lequel les médias actuels fonctionnent. Cependant, à part une étude approfondie sur l'évolution de la radio bretonne au milieu du vingtième siècle (Calvez, 2000), il existe très peu de recherches publiées sur les médias audiovisuels en breton. Il est important de constater que le travail de Stefan Moal (1999, 2001, 2004, 2010) dans ce domaine est pionnier. Intégrant les implications du breton dans les médias sur l'usage de la langue, ainsi que des applications possibles dans l'éducation, Moal présente des aperçus ciblés de la télévision actuelle en breton qui comprennent parfois des éléments comparatifs internationaux.

À part le travail de Calvez sur les émissions de radio et de Moal par rapport aux émissions contemporaines en breton, la plupart des études existantes sur les médias contemporains se veulent plutôt être état des lieux qu'analyse. Elles n'abordent ni le contenu des émissions, ni la problématique de l'utilisation de la langue d'une manière profonde, même si Browne $(1996,2005)$ y a réfléchi. Eithne O'Connell prétend que :

No serious effort to develop minority language media studies as a discipline in its own right, complementing media studies on the one hand and minority language studies on the other, can make significant headway unless it takes a long, detailed look at the specifically linguistic elements of minority language media studies. (O'Connell, 2007 : 227)

Aucun effort sérieux pour développer l'étude des médias en langue minoritaire en tant que discipline à part entière, qui complète d'une part les études des médias et d'autre part les études des langues minoritaires, ne peut faire des avancées significatives sans examiner dans le détail les éléments particulièrement linguistiques de l'étude des médias en langue minoritaire. 
Pour cette raison, cet article est axé sur l'utilisation de la langue pendant le tournage, afin de mieux comprendre quelle incidence ces pratiques peuvent avoir sur l'image de la langue. Avant d'aborder les pratiques des acteurs, il est nécessaire de considérer les paysages sociolinguistique et médiatique dans lesquels la production audiovisuelle a lieu.

\section{Contexte sociolinguistique et médiatique}

Quand on pense à une langue, on pense non seulement à la communication, mais aussi aux richesses culturelles qu'elle héberge. Une langue est à la fois forme et contenu, ce qui pourrait être décrit comme " patrimoine intangible ». Seule, la langue reste « invisible», mais juxtaposée avec d'autres langues, elle endosse une fonction identitaire (Corcoran, 1994 : 62 ; Butler, 2010 ; Mac Aodha Bhuí, dans Butler, 2010). Ceci est le cas des langues minorisées, tel le breton, car même ses locuteurs les plus fidèles restent conscients de la présence d'une autre langue beaucoup plus répandue - le français.

Si la fonction symbolique ou identitaire d'une langue occulte sa fonction communicative, on entre dans une situation bizarre, où les mots deviennent «totems » (Le Pipec). Tel est le cas du breton, de l'irlandais et du māori dans la sphère publique de leurs territoires respectifs. Le poids d'une image régionale, nationale ou ethnique tire ces langues vers un discours purement rhétorique (lipservice), utilisant la langue indigène sur des panneaux routiers, au début des discours publics ou lors des campagnes publicitaires touristiques. Mais toute cette visibilité rhétorique peut cacher une certaine inaudibilité sur le terrain (Fishman, 1997: 194; Nettle \& Romaine, 2000 : 39-40). Où entend-on parler ces langues?

Quand la face publique de la langue devient symbolique plutôt que réelle ou pratique, on s'éloigne de son vrai pouvoir et de son « génie » (Ó Ciardha, 2007). Cela permet aux autorités (et parfois même aux locuteurs) d'éviter les questions délicates concernant la place de la langue dans la société actuelle. Il est plus facile d'imprimer des affiches bilingues que d'entreprendre une politique linguistique de longue haleine qui ferait face aux vrais besoins des locuteurs.

Cependant, ce côté « image » constitue un élément puissant dans l'estime des locuteurs et l'éveil des non-locuteurs. Dans un précé- 
dent article, j'ai exploré l'idée de l'image d'une langue (Lysaght, Bretagne Linguistique $\mathrm{N}^{\circ} 17$ ). Ici, $\mathrm{j}$ 'indique quelques pistes possibles pour de futures recherches.

Que la langue soit normalisée à l'écran (ce qui peut minimiser les inégalités réelles dans la société) ou réifiée comme un objet à observer (ce qui risque d'engendrer une production destinée à sombrer dans les archives sans engager un public contemporain), une langue minorisée dans le contexte d'un paysage audiovisuel dominé par une langue majoritaire se trouve dans une situation délicate, et pour les producteurs et pour le public. Les médias qui diffusent en cette langue s'engagent, volontairement ou pas, dans des enjeux idéologiques.

Bien que seulement environ $4 \%$ des emplois liés à l'utilisation de la langue bretonne aujourd'hui se trouvent dans les médias (Bouroulleg, 2012), il faut reconnaître que l'influence des médias bénéficie d'une portée beaucoup plus importante par rapport à l'image et au développement de la langue actuelle ${ }^{2}$. Cette influence disproportionnée exige une bonne analyse du secteur, afin de voir comment en tirer les meilleurs résultats.

La diffusion web ou télévisuelle rend la langue minorisée plus visible pour plus de gens, et elle élargit la gamme des domaines dans lesquels la langue est parlée (en tournage, lors des réunions de production) et selon lesquels elle est parlée (genres différents). Le contenu et la variété de la langue sont adaptés pour atteindre des publics divers qui vivent une relation plus ou moins engagée avec la langue et sa culture. L'image présentée est celle de la diversité et de l'inclusivité, consciente des correspondances possibles entre la langue dans « le monde réel» et dans «le monde de l'écran». La façon dont la langue est imaginée sur l'écran, même si elle va au-delà des pratiques linguistiques contemporaines, crée un nouveau monde potentiel pour les jeunes publics (Lysaght, 2004). Le pouvoir de la représentation reste entre les mains des diffuseurs et des producteurs indépendants, dont une partie ne sont pas locuteurs de naissance.

2. Un petit exemple de cette influence serait celui des collégiens de Lannilis, qui, ayant gagné une compétition d'écriture de scénario pour la série web Ken Tuch' en 2012, qui a attiré 35 participations (CHEVET, 2012), ont été tellement intéressés par la journée de tournage en studio chez Brezhoweb qu'ils ont choisi de continuer leurs études en breton (BUANNIC, 2012). 
Une familiarité linguistique et culturelle est incontournable pour pouvoir créer des images qui résonnent, et pour contribuer à réaliser le potentiel créatif des communautés auxquelles appartient la langue.

Un fardeau lourd repose sur les médias dans une société où la langue n'est plus une langue de communauté. Ce fardeau est double. D'une part, les médias se retrouvent avec la tâche de représenter la langue et sa culture. Parfois son image à travers les médias peut représenter la seule « preuve » d'existence de la langue et de sa viabilité (Buannic, 2012 ; Fishman, 2001 ; Le Morvan, 2012). Il est important de reconnaître que les médias fonctionnent entremêlés avec d'autres institutions sociales ou publiques, par exemple l'éducation ${ }^{3}$. D'autre part, il existe une pression des militants et des bailleurs de fonds, qui, chacun de leur côté, ont des demandes diverses. Comment les médias peuvent-ils attitrer leurs publics et satisfaire ces demandes ? Pour bien étudier cet aspect, il faut prendre en compte la place des médias publics et privés ou « libres » dans le paysage régional/national/international.

L'industrie audiovisuelle en Bretagne souffre des budgets « très réduits " (Julien, 2012), ce qui entraîne maints soucis. L'approche comparative avec l'Irlande (et la Nouvelle-Zélande de temps en temps) révèle la grande importance d'une volonté politique pour financer la production médiatique (Guyot, 2007). De plus, on voit bien plusieurs défis comparables (l'image d'une langue « traditionnelle » considérée comme ringarde et dépassée, trouver des interlocuteurs, inventer des termes...). Les cultures et contextes sont bien différents, mais les défis de production des textes médiatiques dans une langue minorisée sont semblables dans divers pays ${ }^{4}$.

Bien que la partie «écran » de cette image soit très importante, cet article insiste plus sur les « coulisses ». Il est utile de considérer les pratiques de production comme la partie immergée de l'iceberg et l'émission comme la petite partie visible. Il faut se demander :

3. Yannig Baron le Dihunet y a fait référence, en disant qu'il faut au moins deux des trois éléments pour faire revenir une langue : la famille, l'école et les médias (cité par JuLIEN, 2012).

4. À part ces défis cités, d'autres éléments de comparaison seront structurels, idéologiques, sociolinguistiques et pratiques : l'ampleur d'une volonté politique, les solutions financières, les questions du public, la portée ou la tradition d'un service public, les identités culturelles... 
comment la langue est-elle employée dans la création des émissions médiatiques?

\section{"Beatha teanga í a labhairt" [La vie d'une langue est d'être parlée]}

Il ne suffit pas de faire une langue de représentation pour « faire propre devant le public » (Chapalain, 2012) - il faut que la langue minorisée soit une langue de travail. Pour les trois chaînes de télévision examinées, le côté représentation a été plus valorisé jusqu'ici. Cela peut être une réponse provisoire acceptable à la situation de «manque » linguistique, mais il n'est pas souhaitable que la langue minorisée devienne un maquillage pour le visage d'une émission, tandis que toute la conception et la planification se déroulent en langue majoritaire.

Selon le document Terms of Trade, 1995, de TG4, l'irlandais doit être utilisé lors des enregistrements, mais il n'y a aucune référence de son utilisation sur le plateau ${ }^{5}$. Actuellement de nouvelles initiatives visent à élargir le champ d'utilisation de la langue en production ${ }^{6}$. Dans leur rapport sur la Rautaki Reo [politique linguistique] proposée à Māori Television (novembre 2009), Stephens et Edwards ont dit :

The wannanga is of the view that there is no greater commitment that can be shown in the regeneration of the Māori language than to speak it. (Stephens \& Edwards, 2009)

Le comité considère que l'on ne peut montrer une meilleure preuve de bonne volonté envers la langue māori qu'en la parlant.

Cela veut dire que le personnel et les équipes qui travaillent dans

5. Déanfar an Clár a scannánú nó a fhís-scannánú agus a thaifeadadh i nGaeilge ar choinnioll go bhféadfaidh gnéithe a bheith sa Chlár nach bhfuil i nGaeilge $i$ gcás inar gá sin mar gheall ar chritéir chruthaitheachta an Chláir (TG4, 1995 : 9, section 14.4)./ The Programme shall be filmed or videotaped and recorded in the Irish language provided that the Programme may contain elements which are not in the Irish language where the creative criteria of the Programme reasonably so determine (TG4, $1995: 8$, section 14.4).*

6. Les recommandations pour la couverture de l'Oireachtas 2010 disent que l'irlandais doit être « la langue de la production » ("Bíodh sé san áireamh gur $i$ an Ghaeilge teanga na léirithe seo”) (www.tg4.ie/corp/tair.asp) (consulté le 8/6/2010). 
une institution qui promeut le développement de la langue devraient parler la langue lors de leur travail. Même si TG4 ne formalise pas cette fonction de la chaîne de la même manière, et même si France 3 est plus responsable d'une identité régionale que linguistique, on peut voir que le principe exprimé par Māori Television est important. Ni vue, ni entendue par le grand public, l'utilisation de la langue minorisée « dans les coulisses » est d'une importance majeure pour le projet entier. D'une part, il témoigne de respect et de véritable implication à l'égard de l'ensemble des efforts entrepris. Il contribue également à l'utilisation de la langue dans la communauté, car il fournit un autre lieu ou domaine où la langue est utilisée de manière normale.

\section{Motivation des acteurs}

Pour une large part des locuteurs de l'industrie, produire des émissions comprend bien plus qu'un travail normal (Mac Murchú, 2008 : 159). Ils sont conscients de leur capacité de "voir double» ou de «walk in two worlds» [passer dans deux mondes] (WheokiMane, 2010). Mac Donnacha remarque que TG4 est un service de télédiffusion «a bhfuil pobal dá cuid féin aici » [qui a son propre peuple] (Mac Donnacha, 2008 : 111) - le peuple étant impliqué dans le projet tandis que le public reste à l'écart. Tearepa Kahi (Ngāti Paoa, Waikato), président du groupe des réalisateurs Māori Ngā Aho Whakaari, considère Māori Television comme une partie d'un effort collectif :

On paper, its purpose is to promote reo and tikanga on screen. The reality is Māori Television carries our hopes, dreams, culture, identity and future forward into our whare, kura and industry, everyday [sic], irrespective of who is working inside the building or without... [W]e all share whakapapa. And we all represent different facets of the same kaupapa. (Kahi, 2008)

Sur le papier, sa mission est de promouvoir la langue et la culture sur l'écran. En réalité, Māori Television porte nos espoirs, nos rêves, notre culture, notre identité et notre avenir vers l'avant, vers nos maisons, nos écoles et notre industrie, tous les jours, peu importe qui travaille au sein du service ou à l'extérieur... Nous partageons tous un héritage. Et nous tous représentons des faces diverses du même désir. 
Dans le contexte breton, on retrouve le même sentiment : « Le milieu bretonnant est trop petit pour se disputer ! Nous sommes complémentaires... » (Buannic, cité dans Chevet, 2012). Lionel Buannic, directeur de la société Brezhoweb, est fier que sa mission consiste à « apporter des programmes en breton aux bretonnants » (Buannic, 2012). Il ne s'agit pas d'ignorer le public non-bretonnant, qui reste un groupe à « sensibiliser $~^{7}$, mais de mettre en place d'abord un vrai service pour les locuteurs. Youenn Chapalain, réalisateur et animateur, souligne l'importance de la langue pour son travail : «C'était mon objectif de travailler en breton - on n'est pas crédible quand on reçoit notre public et nos invités sinon » (Chapalain, 2012).

Dans les milieux māori et irlandais on reconnaît les avantages culturels qui découlent de l'utilisation de la langue minorisée comme élément intégral du processus de production :

not just the Màori cultural insight it provides, but the perspective one can only gather when working the stories in one's own language. (Paul, $2005:$ 44)

pas simplement l'aperçu culturel qu'il permet, mais aussi la perspective à laquelle on peut accéder seulement lorsqu'on travaille les sujets dans sa propre langue.

Bien sûr, il faut admettre que la langue ne sera pas regagnée comme moyen de communication du jour au lendemain. Huirangi Waikerepuru, qui jouait un rôle important pendant les campagnes pour un service télévision Māori dans les années 80, explique que la stratégie devrait continuer dans la durée, « maybe 100 years » [peutêtre un siècle] (Waikerepuru, 2009). Le réalisateur de la Gaeltacht Ciarán Ó Cofaigh pense de façon similaire :

Tá tú ag caint ar rud an-fadtéarmach. Ní inniu nó inné a bhéas na rudai seo bainte amach [...] Ní bheidh muid beo chun toradh ar obair TG4 a fheiceáil. Táimid ag iarraidh na céadta bliain de stair a iompú timpeall. (Ó Cofaigh, cité dans Quinn 2009: 15-6)

Vous parlez de quelque chose de longue durée dont les résultats ne seront visibles ni aujourd'hui ni demain [...] Nous ne

7. Buannic voit la stratégie de sensibilisation durer pendant cinq à dix ans (BUANNIC, 2012). 
serons plus vivants pour voir les fruits du travail de TG4. Nous essayons d'aller contre le courant des siècles d'histoire.

\section{Défis liés à la création des émissions en langue minorisée}

Chaque phase de production a ses défis particuliers et quand il s'agit de travailler dans une langue minorisée, la liste de défis s'agrandit.

Pré-production : conception, accès du personnel linguistiquement et techniquement compétent, trouver des invités/comédiens qui parlent bien et qui maîtrisent leur sujet, convaincre les sources financières de la validité du projet.

Production: accès aux fournitures (par exemple, dans un studio partagé avec des productions en langue majoritaire), enjeux techniques et légaux, communication entre les membres de l'équipe ou entre l'équipe et les invités ou même avec le metteur-en-scène, traduction, création de nouveaux termes.

Post-production : communication entre monteur et réalisateur, accès à des réseaux de distribution, attirer et atteindre des publics assez différents, traduction (sous-titrage ou doublage).

Il existe deux groupes confrontés à ces défis : les diffuseurs (chaînes des télévision) et les réalisateurs (sociétés de production). Ces deux groupes répondent à leurs défis selon leurs propres ressources et imaginations.

\section{Les diffuseurs}

À l'échelle d'un diffuseur, il y a des contraintes vis-à-vis des financements, des grilles d'émission et parfois des possibilités d'embauche. Les trois chaînes concernées ont un cahier des charges « service public ». Māori Television en est la seule en Nouvelle-Zélande, ce qui joue en sa faveur au niveau du public, car le service attire un public qui a soif de regarder des émissions de « chez eux », même si elles sont dans une langue qui n'est pas la leur. En Irlande et en Bretagne, les émissions en langue minorisée existent à côté d'autres émissions locales ou régionales en anglais ou en français. Cela réduit l'attirance des productions en irlandais ou en breton, car le public préfère ne pas avoir à « travailler » pour comprendre (Moring, 2007). 
Dans cette situation, il est encore plus important que le contenu et les valeurs visuelles des émissions en langue minorisée soient de haute qualité.

La Bretagne ne dispose pas de son propre service de télévision, mais si on regarde les cas de TG4 (Irlande) et Māori Television (Nouvelle-Zélande), on voit bien que l'existence d'une chaîne ne résout pas tous les problèmes ${ }^{8}$. D'ailleurs, la part d'audience en Bretagne, bien que semblable aux chiffres de Nouvelle-Zélande et de l'Irlande, est considérée comme petite par rapport au territoire français. Cela veut dire que les budgets seront toujours restreints. Dans un cas pareil, l'internet serait peut-être un meilleur moyen de diffusion. Certainement, Brezhoweb en est satisfait, comptant environ 12000 visiteurs You Tube par mois (entre diffusions directes et sur demande) et ils ont 30000 visiteurs par an, plus que d'autres télédiffuseurs traditionnels.

Si TG4 et Māori Television ont été établis pour servir un public qui utilise la langue minorisée (mais nationalement reconnue), France 3 est d'abord un service régional de proximité et peut ensuite se diriger vers des actions pour les langues régionales. La tendance centralisée frustre une vraie relation avec les publics. Par exemple, le studio pour les émissions en breton se situe à Rennes, tandis que la plupart des locuteurs habitent en Basse-Bretagne. Il y a très peu de consultation en ce qui concerne les envies du public quant au contenu des émissions ou leurs créneaux horaires ${ }^{9}$. Les structures institutionnelles des médias français jouent un rôle important sur la réalisation des émissions en breton. Une étude sur l'efficacité de ces façons de travailler sera intéressante à entamer. Par rapport à la position des médias en Irlande et en Nouvelle-Zélande, la situation

8. Les trois chaînes achètent ou commissionnent plus qu'elles ne produisent ellesmêmes, et dépendent des sociétés de production indépendantes pour remplir la plupart de leurs grilles.

9. France 3 est décrit comme contrainte par ses structures internes et par sa place dans un réseau national : "pyramidale... sclérose... cloisonnée » (CHAPALAIN, 2012 ; Daniellou, 2012 ; BuAnNic, 2012 ; Julien, 2012). Un manque d'indépendance en résulte, ce qui peut nuire au bon déroulement du tournage. Par exemple, ils ne peuvent pas toujours choisir leurs équipes en fonction de la langue et se retrouvent obligés de traduire pour des techniciens non-bretonnants (Chapalain, 2012). 
française est particulière, ce qui peut être dû à son histoire d'État centralisateur. Au niveau de la télévision, il semble parfois y avoir une réticence à prendre des risques professionnels de crainte de s'exclure à jamais du seul système qui regroupe les ondes de diffusion.

Māori Television a développé des émissions pour les locuteurs débutants, où les structures et locutions de la langue sont présentées d'une manière agréable (Kōrero Mai, Tōku Reo). Ce genre de pratique sert à sensibiliser un public largement ignorant, mais risque aussi de «cloisonner» la langue, car elle est vue comme but à atteindre plutôt que comme moyen de communication. TG4 se vante de créer de la télévision en irlandais, et non pas pourvoir à l'éducation linguistique. Cette décision de privilégier les locuteurs qui n'ont pas eu de service télévisuel au sens propre du terme avant l'établissement de TG4 en 1996 n'attire pas le large public qui ne parle pas bien l'irlandais. Si la langue est valorisée par une telle approche, elle reste mystérieuse ou moins accessible à la majorité des spectateurs, malgré les sous-titres en anglais. La première chaîne nationale (qui diffuse principalement en anglais) a pu faire une émission qui visait les non-locuteurs directement (Bernard Dunne's Bród Club (Agtel/ Independent Pictures, 2012). L'Irlande a la chance d'avoir plusieurs chaînes nationales qui peuvent utiliser les deux langues (même si en pratique, c'est TG4 qui fait la plupart des productions en irlandais), ce qui permet une certaine complémentarité.

Le fait que les chaînes diffusant des émissions en langue minorisée soient situées dans des contextes nationaux peut parfois leur faire perdre courage. Une solution intéressante à ce problème est l'initiative de Māori Television, qui évite le paysage immédiat pour un univers virtuel beaucoup plus large. Le World Indigenous Television Broadcasters Network (WITBN) [Réseau mondiale de télédiffuseurs indigènes] met en relation des diffuseurs qui privilégient les langues ou les cultures minorisées de par le monde. C'est un réseau international qui partage des émissions, du savoir-faire, et qui organise des échanges de personnel pour des formations (WITBN, 2010).

Pour produire des émissions en langue minorisée dans un contexte de manque (de personnel avec des compétences techniques et linguistiques), on voit deux sortes d'approches. L'une consiste à utiliser des maisons de production établies et à employer un conseiller linguistique ou culturel pour les aider. Souvent, cela a pour ré- 
sultat une version plus ou moins acceptable de la langue diffusée sur l'écran, sans qu'il n'y ait aucune version utilisée « dans les coulisses ». Un vrai progrès implique l'utilisation de la langue pendant le tournage d'une manière naturelle, mais l'histoire sociolinguistique des trois pays veut que cela reste difficile à réaliser. Pour assurer l'utilisation de la langue minorisée pendant toutes les phases de la production, le diffuseur devrait commissionner seulement des maisons « locutrices » (une idée soutenue par les locuteurs, mais pas par les diffuseurs, qui verraient leur pool de sources potentielles d'émissions réduites) ou d'exiger que certains aspects de la production se passent en cette langue (par exemple, les instructions de tournage, les rapports, documents et budgets). Cette approche prescriptive peut être le seul moyen d'aboutir à une utilisation de la langue « dans les coulisses » jusqu'au moment où il existe suffisamment de bons locuteurs avec des compétences techniques à tous les niveaux de l'industrie pour le faire « naturellement ».

Māori Television et TG4 soutiennent l'utilisation de leurs langues respectives en interne, mais ce premier a plus de difficultés que le dernier, car une bonne proportion de son personnel ne parle pas bien te reo et il y a moins de spectateurs avec une compétence passive. La chaîne a développé une politique linguistique particulière pour faire face à ces deux problèmes. La Rautaki Reo [politique linguistique] de Māori Television, conçu entre 2007 et 2010, contient deux éléments : former le personnel afin que les studios soient des environnements « reo » (et tikanga) et atteindre certains niveaux de qualité et de quantité de reo sur l'écran.

Des 120 personnes qui travaillent chez Māori Television, seulement 20 parlent bien te reo Māori (Edwards \& Stephens, $2009: 1)^{10}$. Le but actuel est la «normalisation» de la langue dans le lieu de travail et pour 2024, que $100 \%$ du personnel parlent te reo (Edwards $\&$ Stephens, 2009 : 4). Depuis quelques années, des cours de langue sont proposés au sein de l'entreprise (Berryman, 2007). Une initiative similaire a été essayée à France 3 dans les années 80 , pour que les

10. Si une parfaite maîtrise de la langue n'est pas exigée pour être embauché à Māori Television, "[i]t is important, however, that you are committed to learning, using and fostering te reo Māori» [il est important, cependant, que vous soyez motivé(e) à apprendre, à utiliser et à favoriser la langue māori] (Māori Television website, 2010). 
techniciens améliorent leur breton (Chapalain, 2012). Mais les cours ponctuels ne suffiront pas s'ils ne sont pas accompagnés par une pratique quotidienne.

Quant à ce que l'on voit à l'écran, le niveau de soutien linguistique proposé par le diffuseur varie selon le genre, par exemple chez TG4 il y a un conseiller pour les actualités (coproduites avec RTÉ) (Ó Gallchóir, 2008), tandis qu'il n'y en a pas pour les émissions de divertissement ou de sport $^{11}$. La décision est prise selon le contenu et le genre de l'émission, ainsi que les capacités de l'équipe de production. Pour les productions où le directeur ne parle pas couramment l'irlandais, un comhairleoir teanga [conseiller linguistique] doit être présent. Mais même quand il existe des compétences linguistiques chez le personnel, l'utilisation de la langue « dans les coulisses » dépend en grande partie de la vision de la maison de production (Mac Dhonnagáin, 2010) ${ }^{12}$.

\section{Sociétés de production}

Le paysage audiovisuel des trois langues étudiées est marqué par l'énergie des acteurs qui veulent mettre en œuvre de nouvelles perspectives. Les réalisateurs indépendants se sentent très impliqués dans le développement de leur industrie et expriment souvent leur frustration d'agir sans pouvoir vraiment influencer les structures ou pratiques de leurs commissaires. Plusieurs d'entre eux parlent de l'importance d'utiliser leur langue tout le long du parcours de production.

Actuellement, il est rare pour eux de faire une émission sans avoir recours à la langue majoritaire. Déjà, trouver du personnel n'est pas toujours évident. Buannic constate que parmi les sept candidatures pour le poste d'animateur pour l'émission 123 , il y avait

11. Cela est semblable à la pratique chez Māori Television, où le présence d'un conseiller n'est pas exigée si les réalisateurs ont la compétence de le faire auxmêmes (Evans, 2010).

12. Johnstone cite quelques exemples des fictions récentes sur TG4 : «Aifric mar shampla, bhí Gaeilge ag $99 \%$ den gcriú agus $100 \%$ de na haisteoirí. An Crisis - $20 \%$ den gcriú. Rásaí - 10\% den gcriú [pour Aifric par exemple, 99\% de l'équipe et $100 \%$ des comédiens parlaient irlandais. Pour An Crisis, c'était 20\% de l'équipe et pour Rásai na Gaillimhe environ 10\% de l'équipe] » (JoHnstone, 2010). 
des personnes dont le niveau parlé du breton n'était pas à la hauteur (Buannic, 2012). Parfois il y a des locuteurs doués mais qui manquent de formation technique. Pour l'instant, il n'y a pas de soutien pour former les bretonnants ${ }^{13}$ et cette tâche revient aux petites maisons de production. Le doublage est considéré par Julien de la société Dizale comme un bon moyen de former les comédiens dans un premier temps (Julien, 2012).

Le fait de devoir travailler en deux langues (une pour les techniciens, une autre pour les invités) est « très très fatiguant... vraiment pénible » (Chapalain, 2012) selon ceux qui parlent bien. En plus de leur travail de réalisateur ou animateur, ils doivent se mettre à la disposition des non-locuteurs sur le plateau et agir comme traducteurs. À part la fatigue, il peut y avoir une incidence sur la qualité de l'émission finale. Il est facile, par exemple, d'imaginer les conséquences d'un metteur en scène qui ne maîtrise pas la langue des invités (il resterait sur des gros plans au lieu d'aller de plus près, par exemple).

Les petites sociétés disposent de moins de poids financier, mais ont souvent un lien plus immédiat avec leurs publics. Leurs solutions consistent à être pratiques, par exemple, créer une ambiance où la langue minorisée est la langue de travail (immersion) tout au long du processus de production ou, dans les cas où il n'y a pas suffisamment de bons locuteurs pour faire une équipe entière, mener des pratiques de « cotton-wooling» [mettre du coton autour] (Evans, 2010). Ils essaient de développer leurs idées d'émission dans une perspective « bretonne », « gaélique » ou « māori » comme point de départ.

Combiner reo (la langue) et tikanga (valeurs culturelles) dans l'environnement d'une société de production représente une progression naturelle pour Quinton Hita de Kura Productions :

Running a company is the same as bringing up children. It is a long-term vision. I feel the same way. You have to be very

13. La première politique linguistique en Bretagne date de 2004, par rapport à la loi néo-zélandaise donnant un statut officiel à te reo (1987) et la déclaration du statut national de l'irlandais (1937). Ronan Le Louarn du Conseil régional de Bretagne explique l'ampleur du travail qui reste à faire pour mettre en avant les langues régionales : " on a franchi le plus bas... il y a une place [pour ces langues] qui est extrêmement insuffisante. Tant qu'il n'y a pas de politique générale de l'État, il nous faut une loi pour inciter les gens à se réapproprier leur langue » (LE LOUARN, 2012). 
gentle, and talk... as much as possible. Sometimes I just talk so that they can hear the words, and get used to constructions and phrases - not because I have anything particular to say. (Hita, 2010)

Mener une entreprise est la même chose que d'élever des enfants. C'est une vision à long terme. Pour moi c'est pareil. Il faut être doux et parler... autant que possible. Parfois je parle seulement pour qu'ils entendent les mots et qu'ils s'habituent aux constructions et aux phrases - pas parce que j'ai quoi que ce soit de particulier à dire.

Baigner dans un environnement sonore peut sembler ridicule, mais en fait il est incontournable pour le développement des compétences et pour être à l'aise avec la langue. Quelques maisons de production Māori travaillent seulement en langue māori ou ont rédigé une politique interne reo (par exemple Kura, Māui, Cinco Cine), ce qui leur permet de construire « une architecture culturelle Māori » (Hita, cité dans BERL, 2009 : 20). Si la création de zones linguistiques peut sembler étrange d'emblée, petit à petit le personnel débutant commence à s'y habituer, et développe une confiance et une compétence à parler lui-même cette langue. Utiliser la langue minorisée « dans les coulisses » est une source de fierté pour plusieurs sociétés irlandaises (par exemple Rosg, Scannáin Dobharchú, Telegael) (Ní Bhrádaigh, 2008 : 147) ${ }^{14}$. En Bretagne, on observe la même pratique, où JPL Films, Paris Brest et Vivement lundi utilisent le breton de manière continue (JPL Films, 2014 ; Pois Chiche Films, 2014 ; Paris-Brest Productions, 2014).

Depuis 1997, les trois réalisateurs de la maison de production Kalanna développent des émissions en breton pour la télévision. Leur façon de travailler est de faire le plus possible en breton. Selon Soazig Daniellou, le fait que la langue soit devenue « transparente » a créé une sorte de liberté : « on a pu oublier le breton et se concentrer à faire le film » (2013). Pour Lann $\operatorname{Vras}^{15}$, premier long métrage de

14. Irial Mac Murchú, Directeur général de Nemeton, est fier que l'irlandais soit utilisé à tous les niveaux de l'entreprise, située dans une Gaeltacht : pendant le tournage, pour résoudre des problèmes techniques et dans in production, for technical matters, and in the boardroom (Mac Murchú, 2008 : 157).

15. Le film parle d'une jeune femme, Gwen, qui rentre chez elle, dans sa famille d'ostréiculteurs, après des années en Amérique latine, juste au moment des 
fiction en breton, tout le processus (des ateliers de développement au montage ${ }^{16}$ ) s'est déroulé en breton, ce qui a favorisé une complicité et solidarité entre les membres de l'équipe. Évidemment, cela a aussi rendu les choses plus faciles pour les comédiens.

\section{Le public}

Si l'utilisation de la langue minorisée rend le travail plus évident pour les équipes de tournage, elle aura aussi son influence sur ce que voient les spectateurs. Toute société de production doit rester attentive à ses publics. Kalanna a décidé de faire parler ses personnages en français et en anglais en plus du breton (qui reste majoritaire) pour " ouvrir l'histoire à un public non-bretonnant » et pour présenter un «monde réaliste » (bilingue) (Daniellou, citée dans Nic Giolla Iasachta, 2014).

Quand l'équipe de Lann Vras déjeunait dans un restaurant routier, elle continuait à se parler en breton. Cela a provoqué des réactions positives : « des gens sont venus nous voir, nous dire "ma grand'mère parlait le breton" ou "mes enfants sont scolarisés dans une école bilingue"... Les gens étaient contents d'entendre parler le breton » (Daniellou, citée dans Nic Giolla Iasachta, 2014) ${ }^{17}$.

Samuel Julien explique que le public actuel pour les émissions en breton est majoritairement celui qui a appris la langue. Pour les locuteurs natifs, peu habitués à entendre leur langue parlée par d'autres que leurs proches, la comprendre à la télévision peut parfois exiger un effort même si leur niveau est impeccable (Julien, 2012). Quant à leur opinion sur la qualité du matériel, Julien n'a « jamais eu

tensions avec une association qui protège des sternes. L'expérience de la réalisatrice, Soazig Daniellou, dans le domaine du documentaire laisse des traces et le thème de conflit entre homme et nature est nuancé par les personnages actifs qui sont impliqués dans leur communauté côtière.

16. En 2011, Kalanna a organisé des ateliers de script, avec le soutien de l'écrivain, musicien et éditeur irlandais Tadhg Mac Dhonnagáin, qui a co-créé la série irlandaise jeune public Aifric (actuellement (2011) diffusée doublée sur Tébéo).

17. Pendant le tournage du Foeterien (un jeu télévisé où trois équipes "partent sur les traces de 7 grands personnages de l'histoire de Bretagne » (Gwengolo Filmoù, 2012) en août 2012, et de façon anecdotique, j'ai pu constater les réponses du public par rapport à l'utilisation du breton dans les lieux publics (une vieille personne dans le Tram a spontanément dit : «Ken plijus eo klevet brezhoneg 》 [C'est si agréable d'entendre du breton]). 
de retours négatifs du public bretonnant de naissance » (2012). Avec les dessins animés doublés, Dizale tient à attirer un jeune public, non seulement pour regarder depuis chez eux, mais aussi pour visiter les studios. Les classes sont invitées à voir le breton et la technologie en action à Lorient : « Ils se projettent... ça donne envie aux petits enfants » (Julien, 2012). Si les sociétés de production réussissent à créer des émissions de qualité, leur public potentiel s'élargit. «Avec un bon programme bien fait, on peut dépasser le public "naturel" breton » (Chapalain, 2012).

\section{Technologie}

Le public «naturel» devient un public plus émietté, car les technologies actuelles permettent des changements dans les modes de consommation de télévision. Il n'y a plus besoin d'attendre un créneau particulier, quand on peut accéder aux services vidéo à la demande. Lionel Buannic de Brezhoweb croit à l'avenir des médias de niche : " on a voulu faire une chaîne qui nous ressemble et qui décloisonne la langue bretonne» (2012). À part ses émissions de flux (qui comblent un manque - ce genre n'étant pas éligible aux financements « culturels »), l'équipe de Brezhoweb va sur le terrain deux fois par mois «où les autres ne peuvent pas aller» (2012). Cette présence physique est importante pour montrer au public que les émissions en breton peuvent être près de chez eux et qu'elles ne sont pas emprisonnées dans un studio ni dans une école ${ }^{18}$. Selon Daniellou, si l'importance des services web est claire, il y a toujours besoin de garder une présence à la télévision traditionnelle « pendant encore dix ans au moins... pour que les gens puissent tomber sur un programme en breton » (Daniellou, 2012). Il existe plusieurs publics pour ces émissions et leurs façons d'y accéder pourront changer encore dans quelques années.

18. Brezhoweb a connu un grand succès depuis son début (suite à la fin de TVBreizh). Elle est la première chaîne en langue régionale à devenir conventionnée (CSA). La moitié des conventionnées sont des chaînes généralistes. La société a été contactée par des groupes en Aquitaine et en Occitanie qui cherchent des conseils pour établir leurs propres chaînes web régionales (BUANNIC, 2012). 


\section{Effet sur l'équipe}

L'utilisation de la langue pendant le tournage a aussi des effets intéressants sur l'équipe elle-même. On voit les réalisateurs qui ne parlent pas bien commencer à s'essayer à quelques phrases après des semaines entourées de leurs collègues locuteurs (Lysaght, $2012: 5$ ). $\mathrm{Si}$ au début, l'équipe trouve la présence d'une autre langue nuisible ou agaçante, en général, elle finit par reconnaître la valeur de cet « autre monde » culturel. Un exemple : le court métrage bilingue Te Whare [La Maison] (Green, 2008), conçu dans un respect de tikanga, où chaque jour commençait avec un karakia (prière traditionnelle). Cependant, la langue restait source d'embarras pour le metteuren-scène, qui disait «tak-é one » [prise 1 (l'anglais prononcé à la maori)], jusqu'à ce que la scripte et le deuxième assistant à la caméra aient changé le panneau (slate) en «haerenga tuatahi» [Prise 1 en maori correct].

\section{Conclusion}

Pour les diffuseurs comme les sociétés de production, les nouvelles plateformes (web, portables, etc.) peuvent être utiles pour atteindre leurs publics géographiquement dispersés. Pour le développement du personnel (si le temps et les finances le permettent), ils peuvent organiser des formations internes. Structurellement, il existe plusieurs modèles : une chaîne de service public d'envergure nationale, une chaîne appartenant à un réseau régional ou national, ou une chaîne virtuelle qui diffuse uniquement par internet. Au niveau des finances, apparaissent des initiatives coopératives, comme Oscailt (Irlande) ou Brudañ ha Skignañ (Bretagne) (Lysaght, 2013 : 157).

Quand l'action trouve son origine dans des petites sociétés de production, on observe l'apparition d'une vraie fierté et d'une motivation. Ce genre de pratique, en insistant sur la capacité de la langue dans le monde actuel, met la pression sur les diffuseurs pour fournir du personnel locuteur, des documents bilingues et témoigner plus de respect pour la langue. Ces actions peuvent sembler loin du public, mais on a vu des exemples où les gens sont agréablement surpris d'entendre leur langue ancestrale dans un contexte quotidien.

Pour l'instant, Māori Television est la seule chaîne à développer une politique linguistique pour la bonne utilisation de te reo (depuis 
2009). Cela pourrait éventuellement représenter un modèle pour certains médias en Europe. Il faut donc entreprendre une analyse méthodique des processus de création et de diffusion.

Même avec une politique linguistique, le vrai progrès sur les lieux de production se réalise seulement si de bons locuteurs motivés sont à l'origine du projet. L'idée de Hita selon laquelle «self-sufficient production companies that function as language domains " [des sociétés de production autosuffisantes qui fonctionnent comme des domaines linguistiques] représentent le seul moyen d'avoir une équipe locutrice (Hita, 2009 : 7) est convaincante. Cela dépend de la présence et de la bonne volonté de tous (de la direction aux personnels intermittents du spectacle) ${ }^{19}$. La plupart de ces sociétés de production sont petites, employant entre trois et dix personnes à plein temps et apportant un complément d'équipe aux moments de tournage. Cela veut dire qu'il n'y a pas beaucoup de temps (ni de grands budgets) pour des formations internes. Cependant, plusieurs de ces petites entreprises tiennent à former des locuteurs sur la technique et des techniciens non-locuteurs par rapport aux langues. Ceci montre leur implication. Leur petite taille peut créer une ambiance familiale et propice à l'apprentissage de la langue. En revanche, leur petite taille veut aussi que leurs bons locuteurs ont bien d'autres responsabilités que d'enseigner à leurs collègues, et le développement des contrats de courte durée joue en défaveur du développement d'une compétence linguistique à un niveau profond.

"We don't have all the time we need to help someone get that level of fluency. But we are contributing. » (Hita, 2010)

On n'a pas tout le temps qu'il nous faudrait pour aider

19. Hita raconte son expérience chez Kura Productions : "The first two to three months were really difficult, as people were getting tired. It's tiring to speak a different language all the time if you are not used to it. You have to keep thinking of words and structures. Finding words and phrases to describe the things people do in an office. Even a native speaker did not have the words, e.g. filing cabinet.» [Les deux ou trois premiers mois étaient vraiment difficiles, car les gens se fatiguaient. C'est fatiguant de parler une autre langue en permanence si on n'a pas l'habitude. On doit toujours réfléchir aux mots et aux structures. Trouver les mots et les phrases pour décrire ce qu'on fait dans un bureau. Même un locuteur natif n'aurait pas tous les mots, par exemple, une armoire à archives] (Hita, 2010). 
quelqu'un à atteindre ce niveau de compétence linguistique. Mais on y contribue.

\section{Conclusion générale}

Cet article a examiné trois contextes différents, chacun avec son histoire différente et cadre juridique différent - mais vu la similitude des défis, il semble intéressant d'étudier les stratégies utilisées dans divers pays.

Serait-il possible de partager les savoirs ? La culture télévisuelle est très différente (entre le mode anglophone et francophone, qui forme les environnements qui entourent nos sujets d'étude). Les fonds européens par exemple sont assez compliqués à obtenir si on prend le program MEDIA, on voit que le cadre n'est pas du tout adapté à des langues minoritaires. Il faut alors aller plus loin. Māori Television peut être vue comme une balise (même un phare) dans ce domaine, car ils ont créé l'initiative mondiale WITBN. Pour l'instant, la lingua franca entre les chaînes indigènes reste l'anglais, tout comme dans d'autres réseaux de langues minorisées ${ }^{20}$.

La politique linguistique initiée par Māori Television devrait être suivie, car elle est prometteuse. Le même genre d'action se passe en Bretagne et en Irlande à plus petite échelle, dans les sociétés de production. Il leur faudra plus de soutien pour continuer leur travail.

\section{Recherche restant à mener}

Il faut une étude plus complète de l'utilisation des langues minorisées dans la production $\mathrm{AV}$ actuelle, et l'élément comparatif me semble approprié, car il permet de regrouper les défis et aller vers un échange de solutions, plutôt que de se contenter de constater les problèmes.

Trois parties devraient être examinées :

1. La présence et le poids de la langue dans la conception du projet (décisions prises avant le tournage, choix du personnel, qualité de l'histoire et son développement).

20. Julien (2012) regarde le Festival des Médias Celtiques dans cette optique les émissions bretonnes sont moins nombreuses et moins cotées que celles des autres pays celtiques. Le cercle vicieux de manque de fonds et de différence de cadre culturel peut se traduire en plusieurs langues. 
2. L'utilisation quotidienne de la langue pendant le tournage (relations de proximité avec les comédiens, locuteurs, équipe technique, mélange ou calques avec la langue majoritaire voisine, nécessité de traduire pour certains collègues).

3. Les perspectives crédibles pour l'avenir (besoin d'une politique ou d'un plan linguistique - même minime, pour commencer - adapté aux besoins particulier du secteur, car aller « au fil de l'eau » implique un abandon du gouvernail).

Le but de toute cette recherche est d'inciter à reculer pour pouvoir mieux sauter. Il faut découvrir tout ce qui peut être utile pour arriver à prévoir un avenir plus satisfaisant pour les acteurs et les publics concernés par les médias en langue minorisée.

\section{Bibliographie}

Agtel/ Independent Pictures, « Bernard Dunne’s Bród Club », RTÉ, 2012. BERL Economics, «Māori Television and its contribution to the New Zealand Economy», Report to Māori Television, Wellington, 2009. http://corporate.maoritelevision.com/Default.aspx?tabid=170.

BERRYMAN Charles, «Ngāti Awa - former General Manager Reo and Tikanga, Māori Television - Personal Interview», 2007.

BOUROULLEG Catherine, «Arsellva implij ar yezhoù/ Observatoire des pratiques linguistiques - Emgav/ Entretien », 2012.

BROUDIC Fañch, Parler breton au XXIe siècle : le nouveau sondage de TMO régions, Brest, Emgleo Breiz, 2009.

BROWNE Donald, Electronic media and indigenous peoples : a voice of our own?, 1st ed. Ames, Iowa State University Press, 1996.

Browne Donald, Ethnic minorities, electronic media and the public sphere : a comparative approach, Cresskill N.J., Hampton Press, 2005.

BuANNIC Lionel, « Rener/ Directeur, Brezhoweb - Emgav/ Entretien », 2012.

BUTLER Eoin, « interview with Rónán Mac Aodha Bhuí: 'MC Muppet is a rapper from Connemara whose lyrics are very sexual in content. The Irish language is a very poetic language' », The Irish 
Times, 2010 March 13. http://www.irishtimes.com/newspaper/ magazine/2010/0313/1224265920130.html.

CALVEZ Ronan, La radio en langue bretonne: Roparz Hemon et Pierre-Jakez Hélias : deux rêves de la Bretagne, Rennes, Presses universitaires de Rennes; Brest, Centre de recherche bretonne et celtique, 2000.

CALvez Ronan, « De quoi breton est-il le nom ? », dans Langues de France, langues en danger : aménagement et rôle des linguistes, Délégation générale à la langue française et aux langues de France. Laboratoire Dynamique du Langage (UMR 5596). Laboratoire Interactions, Corpus, Apprentissages, Représentations (UMR 5191). Institut Pierre Gardette. Institut des Sciences de 1'Homme, Université de Lyon, L'Harmattan, 2011.

Chapalain Youenn, «Abadenner, Sevener/Animateur, Réalisateur Emgav/ Entretien », 2012.

CHEvet Brigitte, « Brezhoweb, chaîne en breton sur internet - Actus archivées - Films en Bretagne », accessed 2012 September 26. http://www.filmsenbretagne.com/Actus-archivees-Brezhoweb_chaine-en-breton-sur-internet-1098-445-0-0.html.

CORMACK Michael, and Niamh HourIGAN, Minority Language Media: Concepts, Critiques, and Case Studies, Multilingual Matters, Clevedon, Buffalo, 2007.

DANIELlOU Soazig, «Sevenerez/ Réalisatrice - Emgav/ Entretien », 2012.

EDWARDS Hone \& Tainui STEPHEns, « He Rīpoata nā Tainui rāua ko Hōne/ unpublished report to Māori Television hui on language strategy », Unpublished internal report, Māori Television, 2009.

EVANS Bryn, «Independent producer/director, BraveStar Films Personal Interview », 2010.

FISHMAN Joshua, Can threatened languages be saved?: reversing language shift, revisited: a 21st century perspective, Clevedon, Multilingual Matters, 2001.

GREEN Richard, Te Whare/ The House, Short film, 2008. http:/www. imdb.com/title/tt1527758/.

GuYOT Jacques, « Minority Language Media and the Public Sphere », in Michael Cormack \& Niamh Hourigan ed., Minority Language Media: Concepts, Critiques, and Case Studies, Clevedon, Multilingual Matters, 2007, p. 34-51.

GuYot Jacques, «L'expression médiatique des minorités linguistiques », dans Isabelle Rigoni éd., Qui a peur de la télévision en 
couleurs? : la diversité culturelle dans les médias, Montreuil, Aux lieux d'être, 2007, p. 289-304.

Gwengolo Filmoù, « Gwengolo Filmoù - Degemer - Accueil », accessed 2010 November 15. http://www.gwengolo.com/, 2012.

HITA Quinton, « Personal Interview».

JohnStOne Medb, «Comhairleoir Teanga/ Language consultant Personal e-mail », 2010 May 22.

JPL Films, «JPL Films | Production de films d'animation », accessed 2014 May 30. http://jplfilms.com/.

JULIEN Samuel, « Rener/ Directeur, Dizale - Emgav/ Entretien », 2012.

KAHI Tearepa, "Message from our Chairman », Ngā Aho Whakaari e-pānui/newsletter, December 2008.

Kelly-Holmes Helen, Minority Language Broadcasting: Breton and Irish, Clevedon, Multilingual Matters, 2001.

LE COADIC Ronan, "Langue et modernité », dans Bretagne, le fruit défendu, Rennes, Presses universitaires de Rennes, 2002.

LE LOUARN Ronan, « Chef de service des langues de Bretagne, Rannvro Breizh/ Région Bretagne - Emgav/ Entretien », 2012.

LE MoRVAN Muriel, « Kazetennerez, Abadennerez/ Journaliste, Présentatrice, France 3 Ouest - Emgav/ Entretien », 2012.

LE PIPEC Erwan, 2012. «Le breton, langue-totem?», La Bretagne linguistique, Brest, UBO-CRBC, 2013, vol. 18, p. 137-176.

LYSAGHT Ruth, " Pobal Sobail: Ros na Rún, TG4 and Reality », in Ruth Barton and Harvey O'Brien ed., Keeping it Real: Irish film and television, London, New York, Wallflower Press, 2004, p. 147-158.

LYSAGHT Ruth, "'Show your Bród!' Non-Fluent Speakers in Minority Language Media - Changing the Rules », FEL XVI- AUT, 2012.

LYSAGHT Ruth, «L'image des langues minoritaires à travers les médias contemporains », La Bretagne Linguistique, Brest, UBO-CRBC, 2012, vol. 17, p. 233-252.

MAC DHONNAGÁIN Tadhg, "Skype Interview - Writer », 2010.

MAC DONNACHA Seosamh, «Seirbhís Chraolacháin nó Seirbhís Phleanála Teanga? », in Eithne O'Connell, John Walsh, and Gearóid Denvir ed., TG4@10: deich mbliana de TG4/ten years of TG4, Indreabhán, Cló Iar-Chonnachta, 2008, p. 103-116.

MAC MURChÚ Irial, "Teilifís, Tionscal agus Teanga», in Eithne O'Connell, John Walsh, and Gearóid Denvir ed., TG4 @ 10: deich mbliana de TG4/ten years of TG4, Indreabhán, Cló Iar-Chonnachta, 2008, p. 167-161. 
MOAL Stefan, «Les langues minorisées à la télévision : convergences, divergences, problématiques transfrontalières dans l'Espace Atlantique européen », dans Yann Bevant, Gwendal Denis, Hervé Bihan, Cultures, langues et imaginaires de l'arc Atlantique, Centre d'études irlandaises (Rennes) \& Bretagne-Études celtiques (Rennes), TIR, Publication du CRBC Rennes 2, 2010, p. 199-228.

MOAL Stefan, "Utiliser les infos en breton dans l'enseignement bilingue », dans Francis Favereau éd., Klask: actes du colloque international de Plésidy (Côtes d'Armor), octobre 1997, Klask, 5, 1999, p. 159-168.

MOAL Stefan, "L'anglais et l'irlandais sur RTE et TG4, le français et le breton sur France 3 et TV Breizh », dans Anne Goarzin éd., Bretagne et Irlande, Klask 9, Rennes, Presses universitaires de Rennes, 2004, p. 11-25.

MORING Tom, "Functional Completeness in Minority Language Media », in Mike Cormack and Niamh Hourigan ed., Minority Language Media: Concepts, Critiques, and Case Studies, Clevedon, Buffalo, Multilingual Matters, 2007, p. 17-33.

Ní BHRÁDAIGH Emer, «An Léargas Fiontraíochta », in Eithne O’Connell, John Walsh, and Gearóid Denvir ed.,TG4@10: deich mbliana de TG4/ ten years of TG4, Indreabhán, Cló Iar-Chonnachta, 2008, p. 137-154.

Nic Giolla Iasachta Ruth, «Beo! - Lann Vras, Scannán Nua i mBriotáinis », accessed 2014 May 30. http://www.beo.ie/alt-lannvras-scannan-nua-i-mbriotainis.aspx.

O'ConNell Eithne, «Translation and Minority Language Media: Potential and Problems: An Irish Perspective », in Mike Cormack and Niamh Hourigan ed., Minority Language Media: Concepts, Critiques, and Case Studies, Clevedon, Buffalo, Multilingual Matters, 2007, p. 212-228.

Paris-Brest Productions, " Paris-Brest Productions », accessed 2014 May 30. http://www.parisbrestproductions.com/.

Pois Chiche Films, «Accueil | Pois Chiche Films », accessed 2014 May 30. http://www.poischichefilms.com/.

QUINN Declan, «Athshamhlú na teanga i scannánaíocht na Gaeilge: Ros na Rún, an Gearrscannánaíocht, Aifric, Kings agus The Running Mate », Unpublished togra taighde - final year undergraduate research project, NUI Galway, 2009. 
RIGONI Isabelle, «Introduction», dans Isabelle Rigoni éd., Qui a peur de la télévision en couleurs? : la diversité culturelle dans les médias, Montreuil, Aux lieux d'être, 2007, p. 17-35.

SCHUSTER Manutai, «Tumuaki Kohinga Whakaata/ Head of Acquisitions \& Commissioning, Māori Television - Personal Interview », 2010.

WAIKEREPURU Huirangi, "Contribution », presented at the Māori Television Rautaki Reo hui/ language strategy meeting, Auckland, 2009 November 20.

WHEOKI-MANE Aroha, « Broadcast professional - Personal interview», 2010.

WITBN, «Welcome to the World Indigenous Television Broadcasters Network », accessed 2010 August 24. http://www.witbn.org/. 